\title{
Exploring the Impact of the COVID-19 Quarantine on the Severity of Headache, Migraine, and Stress in Saudi Arabia
}

\author{
Nouran A Aleyeidi (iD) \\ Raneem S Alqahtani ${ }^{2}$ \\ Hawazin F Alotaibi ${ }^{2}$ \\ Amjad H Alotaibi ${ }^{2}$ \\ Kholoud Mohsen Alotaibi ${ }^{2}$ \\ Reenad Mohammed Alnofiey ${ }^{2}$ \\ 'Department of Clinical Sciences, \\ Community Medicine Course, College of \\ Medicine, Princess Nourah Bint \\ Abdulrahman University, Riyadh, Saudi \\ Arabia; ${ }^{2}$ College of Medicine, Princess \\ Nourah Bint Abdulrahman University, \\ Riyadh, Saudi Arabia
}

Correspondence: Nouran A Aleyeidi $\mathrm{Tel}+966504304577$

Email naaleyeidi@pnu.edu.sa
Purpose: This cross-sectional study aimed to investigate the impact of COVID-19 quarantine on the severity of migraine symptoms and stress among adults in Saudi Arabia.

Patients and Methods: Between December 2020 and February 2021, 1212 participants aged 18-65 years completed an online self-administered questionnaire that covered sociodemographic data, self-administered questions, the ID migraine screener, numeric pain rating scale, and the perceived stress scale. Statistical analyses were performed using SPSS. Student's $t$-test, paired $t$-test, and analysis of variance were used to compare quantitative variables, while the chi-square test was used to compare qualitative variables.

Results: After removing ineligible and incomplete responses, we analyzed data obtained from 1111 participants. The mean age of the participants was approximately 29 years old $( \pm$ 11.2 years); moreover, $87 \%$ were females. Headache severity during the COVID-19 quarantine was significantly lower than that during the last 3 months, with a difference of only 0.41 on the $1-10$ pain severity scale. There was a significantly increased stress prevalence during the COVID-19 quarantine. During the COVID-19 quarantine, $49 \%, 56 \%$, and $62 \%$ of patients without migraine, patients with possible migraine, and patients with diagnosed migraine, respectively, reported worsening of their perceived stress. There was a weak positive correlation between the severity of migraine symptoms and stress during COVID19 quarantine. Moreover, headache severity was positively affected by the history of COVID-19 infections.

Conclusion: The headache severity in patients with migraine decreased during COVID-19 quarantine compared with that during the last 3 months. Additionally, patients with diagnosed migraine had significantly higher scores on the perceived stress scale than patients without migraine.

Keywords: migraine, COVID-19, stress, headache, lockdown, quarantine, Saudi

\section{Introduction}

On March 11, 2020, the World Health Organization declared coronavirus disease (COVID-19) a global pandemic. ${ }^{1}$ COVID-19 is a highly contagious disease that spreads among humans through the same transmission mode as other common cold or flu viruses-ie, face-to-face contact with coughs or sneezes or contact with secretions from infected people. ${ }^{2}$ By October 15, 2021, there were more than 239 million confirmed cases worldwide, with approximately 5 million deaths. ${ }^{3}$

In Saudi Arabia, there were more than 547 thousand COVID-19 cases and over 8000 deaths in October 2021. ${ }^{3}$ Many countries worldwide had to enforce lockdowns. Riyadh, which is the capital of Saudi Arabia, was among the major cities to 
implement lockdown or quarantine between the beginning of March 2020 and 21 June 2020. During this period, strict governmental regulations were implemented in Saudi Arabia to control the spread of the disease which included closing schools, universities, malls, restaurants, shops.etc and limiting people's movement by curfew and allowing them to go out from their homes only during limited times.

Quarantine is associated with several adverse psychological effects. During the COVID-19 outbreak, several people suffered from confusion, post-traumatic stress symptoms, and anger. ${ }^{1}$ Stress is the body's response to physical, mental, or emotional pressure. It can cause feelings of anger, anxiety, frustration, or depression. Stress may be caused by normal life activities or incidents such as trauma or disease. High stress levels can contribute to mental and physical health issues. ${ }^{4}$ According to The International Classification of Headache Disorders published in 2018 ICHD-3, migraine is a common disabling primary headache disorder. It's classified into two major types; migraine without aura and migraine with aura. ${ }^{5}$

Migraine attacks rarely occur spontaneously without precipitating factors. The most common precipitating factors for migraine with and without aura are stress and mental tension. Other precipitating factors include not eating at the right times, noise, lack of sleep, menstruation, weather changes, smells, and smoking. ${ }^{6}$ Worldwide, migraine affects 1 in every 10 people. ${ }^{7}$

Moreover, the estimated lifetime prevalence of migraine is $12 \%-18 \%{ }^{8}$

During the COVID-19 outbreak, there was an increase in the prevalence of psychological problems due to quarantine and the stress of infection. ${ }^{9}$ However, there has been scarce research on the effect of quarantine on patients with migraine. A study conducted in Southwest China reported that patients with migraine had extremely high psychological distress during the lockdown compared with healthy controls. ${ }^{7}$

An Italian study published in August 2020 reported a reduced headache frequency and intensity in adult patients with migraine during the quarantine compared with before the quarantine. Moreover, it reported a correlation between headache improvement and the number of days of stay-at-home. ${ }^{1}$ Another Italian study published on May 22, 2020, reported decreased migraine attacks and pain in patients with migraine during the quarantine regardless of moderate depression levels. ${ }^{10}$ Moreover, a recent study conducted in Kuwait City and published in September 2020 reported worsened headaches in patients with migraine during the COVID-19 pandemic. ${ }^{11}$ In the other hand, a study published in
February 2021 and conducted in Spain, had different findings. They used a web-based questionnaire which was distributed on migraine patients to assess their clinical course during COVID19 lockdown. They analyzed 222 responses which showed worsening of usual migraine pain during lockdown in $47.3 \%$ of them. This was positively related to post-traumatic stress, older age and living with five or more people. ${ }^{12}$

In Saudi Arabia, a study conducted at Al Qassim University on 1160 participants reported that $23.6 \%$, $28.3 \%, 24 \%$, and $22.3 \%$ of the patients presented with moderate-to-severe psychological symptoms and moderate-to-severe depressive symptoms, anxiety symptoms, and stress symptoms, respectively, after the outbreak. ${ }^{13}$ However, this study did not address migraine and its relation to stress during the COVID-19 quarantine.

The relationships among migraine, stress, and the lockdown remain unclear. There have been no Saudi Arabian studies on the effect of the COVID-19 quarantine on migraine and stress among adults. Therefore, we aimed to investigate the impact of the COVID-19 quarantine on the severity of migraine symptoms and stress among adults in Saudi Arabia between March 2020 and 21 June 2020, as well as the relationship between migraine and stress during the quarantine period.

\section{Materials and Methods}

This cross-sectional study enrolled participants using convenience sampling via an online survey link made using a free open access Redcap form. The link was shared through a number of social media platforms (WhatsApp, Telegram and Twitter), in two languages (Arabic and English). We included adults aged 18-65 years who experienced the COVID-19 quarantine in Saudi Arabia between March 2020 and 21 June 2020.

This study administered a self-reported survey comprising sociodemographic variables, a migraine screening validated scale (the three-item ID Migraine screener), and assessment of headache severity using the validated numeric rating scale (NRS). Moreover, it included questions regarding the frequency and pattern of headache attacks, questions specific to the COVID-19 lockdown, and the validated Perceived Stress Scale (PSS) for stress assessment before and during the COVID-19 quarantine.

Specifically, we used the Arabic-translated ID-Migraine screener, which is comprised of three "yes" or "no" screening questions. ${ }^{14}$ Additionally, we used the NRS developed by the International Association for the Study of Pain to assess headache severity on a $0-10$ scale, where 0 and 10 indicate no and maximum headache pain, respectively. ${ }^{15}$ Moreover, 


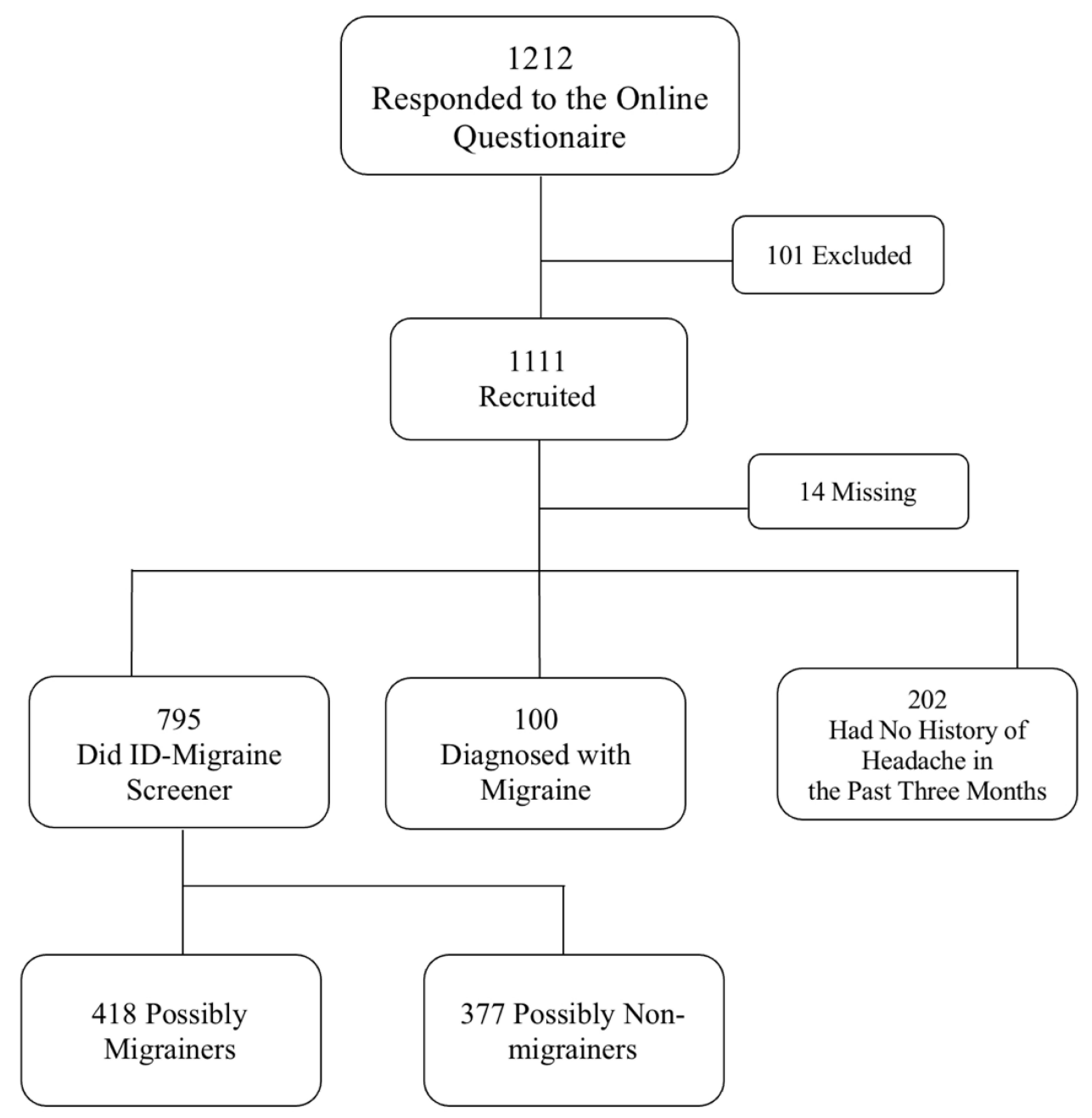

Figure I Flowchart of the study participants depicting their distribution.

we administered a validated Arabic version of the scale. ${ }^{16}$ Stress levels were evaluated using the 10-item PSS (PSS-10), which investigated feelings and thoughts experienced during the last month compared with those experienced during the COVID-19 quarantine. This scale has a validated Arabic version used for Arabic respondents. ${ }^{17}$

Based on a confidence level of $95 \%$ and a $5 \%$ margin error, we calculated the sample size to be 1028 participants based on the result of a previous study where $59.6 \%$ of the participants reported an increase in migraine frequency during lockdown, ${ }^{11}$ with $10 \%$ addition to compensate for incomplete data since this was an online-questionnairebased study. The mean, standard deviation, and percentages were used for descriptive statistics. A $t$-test and chi-square test were used to analyze quantitative and qualitative variables, respectively. Statistical significance was set at $\mathrm{P}<$ 0.05 . The software used for statistical analysis was IBM SPSS Statistics version 25.

\section{Results}

A total of 1212 individuals responded to the online questionnaire. After excluding ineligible individuals, we included 1111 participants in the final analysis (Figure 1). The mean age was $29 \pm 11.2$ years, with females accounting for $87 \%$ of the participants. Moreover, 95\% of the participants were Saudis and 57\% were Riyadh residents. Overall, approximately two-thirds of the study population were single. Degree holders represented $70 \%$ of the whole sample. Employed and unemployed (including housewives and retirees) accounted for $24 \%$ and $16 \%$ of the participants, respectively. Less than $20 \%$ of the participants were obligated to attend to the workplace during the COVID-19 quarantine. Moreover, $15 \%$ of the participants were diagnosed with COVID-19, which was much lower than the expected values (Table 1).

Compared with all participants, participants diagnosed with COVID-19 showed a significantly higher headache 
Table I Characteristics of the Study Sample $(\mathrm{n}=$ IIII)

\begin{tabular}{|c|c|}
\hline Item & Statistics \\
\hline Age (years) & $28.7 \pm 11.2$ \\
\hline \multicolumn{2}{|l|}{ Gender } \\
\hline Female & $962(86.6 \%)$ \\
\hline \multicolumn{2}{|l|}{ Nationality } \\
\hline Saudi & $1056(95 \%)$ \\
\hline \multicolumn{2}{|l|}{ Current Residency } \\
\hline Riyadh city & $636(57.2 \%)$ \\
\hline Outside Riyadh city & $469(42.2 \%)$ \\
\hline \multicolumn{2}{|l|}{ Marital Status } \\
\hline School & $34 I(30.7 \%)$ \\
\hline Bachelor degree & $702(63.2 \%)$ \\
\hline Postgraduate degree & $64(5.8 \%)$ \\
\hline \multicolumn{2}{|l|}{ Occupational status } \\
\hline Student & $524(47.2 \%)$ \\
\hline Full-time employed & $217(19.5 \%)$ \\
\hline Part-time employed & $33(3.0 \%)$ \\
\hline Self-employed & $15(1.4 \%)$ \\
\hline Retired & $28(2.5 \%)$ \\
\hline Unemployed & $151(13.6 \%)$ \\
\hline Housewife & $142(12.8 \%)$ \\
\hline $\begin{array}{l}\text { Working in a job that requires } \\
\text { attendance to workplace during the } \\
\text { COVID-19 quarantine }\end{array}$ & $203(18.6 \%)$ \\
\hline Diagnosed with COVID-I9 & 161 (14.5\%) \\
\hline
\end{tabular}

Note: Values in statistics are presented as frequency (percentage). Abbreviation: COVID-19, coronavirus disease.

severity during the COVID-19 quarantine. There were no other factors affecting the headache severity in the entire study sample. Among patients without migraine, the education level and occupation showed a significant effect on the headache severity during the COVID-19 quarantine. Specifically, the highest headache severity during the COVID-19 quarantine was observed among participants with school as their highest educational level and housewives.

Approximately one-third of the participants suffered headache attacks once a month over the past three months, with few participants suffering from daily headaches.
Table 2 Prevalence and Severity of Headache and Stress in the Study Sample $(\mathrm{n}=\mathrm{I} \mathrm{I} \mathrm{I})$

\begin{tabular}{|c|c|}
\hline Item & Statistics \\
\hline \multicolumn{2}{|c|}{$\begin{array}{l}\text { How frequently did you suffer from headache over the last } 3 \\
\text { months? }\end{array}$} \\
\hline Daily & $43(3.9 \%)$ \\
\hline Once weekly & 225 (20.3\%) \\
\hline Two times or three times per week & $270(24.3 \%)$ \\
\hline Once monthly & 369 (33.2\%) \\
\hline Never & $202(18.2 \%)$ \\
\hline Previously diagnosed with migraine & $100(9 \%)$ \\
\hline $\begin{array}{l}\text { Possible migraineur according to the ID } \\
\text { Migraine screener }\end{array}$ & $418(37.6 \%)$ \\
\hline \multicolumn{2}{|l|}{ Factors worsening the headache } \\
\hline Stress & 521 (46.9\%) \\
\hline Not eating at right times & $263(23.7 \%)$ \\
\hline Noise & 381 (34.3\%) \\
\hline Lack of sleep & $653(68.8 \%)$ \\
\hline Menstruation & 277 (24.9\%) \\
\hline Change in weather & 128 (II.5\%) \\
\hline Smoking & $28(2.5 \%)$ \\
\hline \multicolumn{2}{|l|}{ Perceived Stress Scale results } \\
\hline Mild stress & $9(0.8 \%)$ \\
\hline Moderate stress & $184(16.6 \%)$ \\
\hline High stress & 872 (78.5\%) \\
\hline
\end{tabular}

Note: Values in statistics are presented as frequency (percentage).

Furthermore, one-tenth of the participants reported being previously diagnosed with migraine by a neurologist. After excluding patients with diagnosed migraine and participants without headache attacks in the past three months, the ID migraine screening test was applied for the remaining sample, which showed that patients with and without possible migraine comprised $38 \%$ and $34 \%$ of the total sample, respectively. Headache triggers varied among the participants, with lack of sleep (69\%) and stress (47\%) being the most frequently reported headache triggers. Regarding the stress severity, over the past 3 months, $79 \%$ of the participants showed high stress levels on the PSS (Table 2). 
Table 3 The Effect of the COVID-19 Quarantine on Headache Severity $(n=11 \mathrm{II})$

\begin{tabular}{|c|c|c|c|c|}
\hline \multirow[t]{2}{*}{ Item } & \multicolumn{4}{|l|}{ Statistics } \\
\hline & $\begin{array}{l}\text { Whole Study } \\
\text { Sample (n = } \\
\text { IIII) }\end{array}$ & $\begin{array}{l}\text { Possibly Healthy } \\
\text { Participants } \\
\text { According to the ID } \\
\text { Migraine Screener } \\
(n=377)\end{array}$ & $\begin{array}{l}\text { Participants with } \\
\text { Possible Migraine } \\
\text { According to the ID } \\
\text { Migraine Screener (n } \\
=418 \text { ) }\end{array}$ & $\begin{array}{l}\text { Participants } \\
\text { Diagnosed } \\
\text { with } \\
\text { Migraine (n } \\
=(00)\end{array}$ \\
\hline $\begin{array}{l}\text { Headache severity over the last } 3 \text { months (on a scale } \\
\text { from } 0 \text { to } 10 \text { ) }\end{array}$ & $4.84 \pm 2.18$ & $3.92 \pm 1.88$ & $5.28 \pm 2.03$ & $6.44 \pm 2.39$ \\
\hline $\begin{array}{l}\text { Headache severity during the COVID-19 quarantine (on } \\
\text { a scale from } 0 \text { to } 10 \text { ) }\end{array}$ & $4.29 \pm 2.61$ & $3.23 \pm 2.22$ & $4.91 \pm 2.59$ & $5.66 \pm 2.53$ \\
\hline P value* & $<0.001$ & $<0.001$ & $<0.001$ & $<0.001$ \\
\hline
\end{tabular}

Notes: Values in statistics are presented as: mean \pm standard deviation. *P value is calculated using paired $t$-test, where values less than 0.05 are significant. Abbreviation: COVID-19, coronavirus disease.

The headache severity during the COVID-19 quarantine was significantly lower than that over the last 3 months. It decreased by $0.6,0.7,0.4$, and 0.8 among all participants, possibly healthy participants, patients with possible migraine, and patients diagnosed with migraine, respectively (Table 3 ).

Among the 100 patients diagnosed with migraine, only $23 \%$ reported communicating with their physicians during the COVID-19 quarantine due to an increased headache severity. Moreover, $32.7 \%$ and $6.3 \%$ of the patients reported requiring to visit the emergency department after and during the COVID-19 quarantine, respectively. Approximately $36.7 \%$ of patients were taking prophylactic migraine treatments. Among them, 56.6\% of the participants did not change their usage of prophylactics after the start of COVID-19 quarantine. Moreover, $47 \%$ of the participants reported increasing the usage of acute medications for migraine during the quarantine (Table 4).

During quarantine, stress worsening was reported by $56 \%, 62 \%, 52 \%$, and $49 \%$ of patients with possible migraine, patients diagnosed with migraine, the whole sample, and possibly healthy participants, respectively. (Table 5). There was a weak positive correlation between the severity of migraine symptoms and stress during the COVID-19 quarantine (Pearson's correlation coefficient $=$ $0.224, \mathrm{p}<0.001$ ) (Figure 2).

\section{Discussion}

In this study, patients with diagnosed and possible migraine showed significantly less severe headache during the COVID-19 quarantine than healthy individuals; however, the difference was not clinically significant. Our findings are consistent with previous reports. For example, an Italian study conducted by Delussi et al reported a reduction in the average number of days with headache, days with acute medication intake, and migraine intensity during the quarantine than before the quarantine. ${ }^{1}$ Another Italian study conducted in Genova reported reduced migraine symptoms among migraine patients based on the Global Assessment of Migraine Severity $(5.61 \pm 0.76$ vs $4.16 \pm 1.46 ; \mathrm{p}<0.001)$ and visual analog scale $(7.49 \pm$ 1.10 vs $5.47 \pm 1.88 ; p<0.001)$ scores. ${ }^{10}$ However our results were inconsistent with the study conducted in Spain in February 2021, where they found that $47.3 \%$ of migraine patients reported worsening of their migraine pain during lockdown. ${ }^{12}$

In our study, most of the patients diagnosed with migraine did not require increased use of migraine treatments during the COVID-19 quarantine. However, 23\% of them had to communicate with their physician due to an increased headache severity, while approximately $33 \%$ of them reported admission or ER visit due to increased headache severity during the COVID-19 quarantine.

Another Italian study conducted in Genova, Italy, ${ }^{10}$ reported a reduced weekly frequency of triptan usage for migraine attacks during quarantine than before quarantine (2.00 [1.78] vs 2.84 [2.54]; $p=0.041)$, which is consistent with our findings. The between-period difference could be attributed to the absence of work-derived stress triggers. Additionally, an Italian study conducted by Delussi et al 
Table 4 The Effect of the COVID-I9 Quarantine on the Severity of Migraine Symptoms $(n=100)$

\begin{tabular}{|c|c|}
\hline \multicolumn{2}{|c|}{$\begin{array}{l}\text { Communication with their physicians during the COVID-19 } \\
\text { quarantine due to increased headache severity }(n=100)\end{array}$} \\
\hline Communicate & $23(23 \%)$ \\
\hline Did not communicate & $77(77 \%)$ \\
\hline \multicolumn{2}{|c|}{ Visiting emergency department due to headache $(n=100)$} \\
\hline \multicolumn{2}{|l|}{$32(32.7 \%)$} \\
\hline \multicolumn{2}{|c|}{$\begin{array}{l}\text { Visiting emergency department more than usual during } \\
\text { the COVID-19 quarantine }(n=100)\end{array}$} \\
\hline \multicolumn{2}{|l|}{$2(6.3 \%)$} \\
\hline \multicolumn{2}{|c|}{$\begin{array}{l}\text { Prescription of prophylactic medications for migraine from } \\
\text { a neurology clinic }(n=100)\end{array}$} \\
\hline \multicolumn{2}{|l|}{$36(36.7 \%)$} \\
\hline \multicolumn{2}{|c|}{$\begin{array}{l}\text { Impact of the COVID-I9 quarantine on usage of } \\
\text { prophylactic medications for migraine }(n=36)\end{array}$} \\
\hline Increased significantly & $5(13.9 \%)$ \\
\hline Increased slightly & $5(13.9 \%)$ \\
\hline Did not change & $20(55.6 \%)$ \\
\hline Decreased slightly & $3(8.3 \%)$ \\
\hline Decreased significantly & $3(8.3 \%)$ \\
\hline \multicolumn{2}{|c|}{$\begin{array}{l}\text { The impact of the COVID-19 quarantine on usage of the } \\
\text { acute medications of migraine }(n=36)\end{array}$} \\
\hline Increased significantly & $6(16.7 \%)$ \\
\hline Increased slightly & II (30.6\%) \\
\hline Did not change & $12(33.3 \%)$ \\
\hline Decreased slightly & $4(11.1 \%)$ \\
\hline Decreased significantly & $3(8.3 \%)$ \\
\hline
\end{tabular}

Note: Values in statistics are presented as frequency (percentage). Abbreviation: COVID-19, coronavirus disease.

reported that among 289 patients who took preventive treatments for migraine, 88 discontinued treatment for different reasons, including drug failure or difficulty in accessing drugs. ${ }^{1}$ Another web-based survey conducted in Kuwait city ${ }^{11}$ revealed considerable negative effects of the COVID-19 pandemic on patients with migraine. For example, most patients with migraine (597 [58.7\%]) overused analgesics and acute migraine treatments during the COVID-19 quarantine. Moreover, only $22.5 \%$ of the participants visited the emergency department for acute management. Furthermore, most patients with migraine (626 [61.5\%]) did not communicate with their physicians during the lockdown, which is consistent with our findings. However, patients who communicated with the physicians (136 [13.4\%]) reported visiting the hospital.

In our study, headache severity was positively affected by a history of COVID-19 infection. Among patients with migraine, headache severity was affected by the education levels and occupation. In the Kuwaiti study, ${ }^{11}$ migraine severity was significantly associated with age, occupation, education, and marital status. Moreover, most of the patients with migraine showed worsening symptoms during the COVID-19 quarantine, which could be attributed to infection since 41 (4\%) patients were infected with the virus.

More than half of the participants with diagnosed or possible migraine reported increased perceived stress during the last month and the COVID-19 quarantine. However, in the whole study sample, there was an improvement in their stress level after the quarantine period. Additionally, the PSS score showed a weak positive correlation with headache severity. There is a known relationship between stress and headache. According to Mayo clinic, ${ }^{18}$ the most common headache type is tension headache, with the most common trigger being stress. However, the exact cause of tension headache remains unclear. Moreover, a review of the relationship between stress and headache reported that despite the scarcity of definitive evidence, there is strong evidence indicating that stress can trigger headaches. ${ }^{19}$ Also, there is a study conducted on migraine during COVID-19 lockdown found that the delay in access to their medications during that period caused worsening of their symptoms. ${ }^{20}$ We did not ask about change in access to medications in the current study.

This study has several limitations. First, we used the non-probability convenience sampling technique. Second, most of our participants were females and the older population was underrepresented, which limits the generalizability of our results. Finally, our study involved an online self-reported questionnaire, with the responses being prone to recall difficulties given the relatively long duration of the time window between the end of the quarantine and the administration of our questionnaire, which was about seven months. 
Table 5 The Effect of the COVID-19 Quarantine on the Severity of Perceived Stress $(\mathrm{n}=\mathrm{II}$ II)

\begin{tabular}{|l|l|l|l|l|}
\hline \multirow{2}{*}{ Item } & \multicolumn{2}{|l|}{ Statistics } \\
\cline { 2 - 5 } & $\begin{array}{l}\text { Whole Study } \\
\text { Sample (n = } \\
\text { IIII) }\end{array}$ & $\begin{array}{l}\text { Possibly Healthy } \\
\text { Participants According } \\
\text { to the ID Migraine } \\
\text { Screener (n= 377) }\end{array}$ & $\begin{array}{l}\text { Participants with } \\
\text { Possible Migraine } \\
\text { According to the ID } \\
\text { Migraine Screener (n } \\
=418)\end{array}$ & $\begin{array}{l}\text { Participants } \\
\text { Diagnosed } \\
\text { with Migraine } \\
(\mathbf{n}=\mathbf{~ I 0 0 )}\end{array}$ \\
\hline Reported worsening of stress during quarantine & $577(52 \%)$ & $186(49.3 \%)$ & $233(55.8 \%)$ & $62(62 \%)$ \\
\hline $\begin{array}{l}\text { Reported improvement in stress after } \\
\text { quarantine }\end{array}$ & $758(68.2 \%)$ & $269(71.3 \%)$ & $292(69.9 \%)$ & $76(76 \%)$ \\
\hline High stress on the Perceived Stress Scale & $872(78.5 \%)$ & $282(43.9 \%)$ & $360(56.1 \%)$ & $84(84 \%)$ \\
\hline
\end{tabular}

Abbreviation: COVID-19, coronavirus disease.

\section{Conclusion}

In conclusion, our study showed that stress and sleep disturbance were the most reported triggers for headache. Moreover, headache severity in patients with migraine decreased during the COVID-19 quarantine than during the last 3 months. Additionally, patients diagnosed with migraine had significantly higher PSS scores than possibly healthy participants. Headache severity was positively affected by a history of COVID-19 infection. Future studies should include more males and geriatric participants. Furthermore, future studies should recruit more participants from the neurological clinics of different hospitals in Saudi Arabia to increase the responses of patients with migraine.

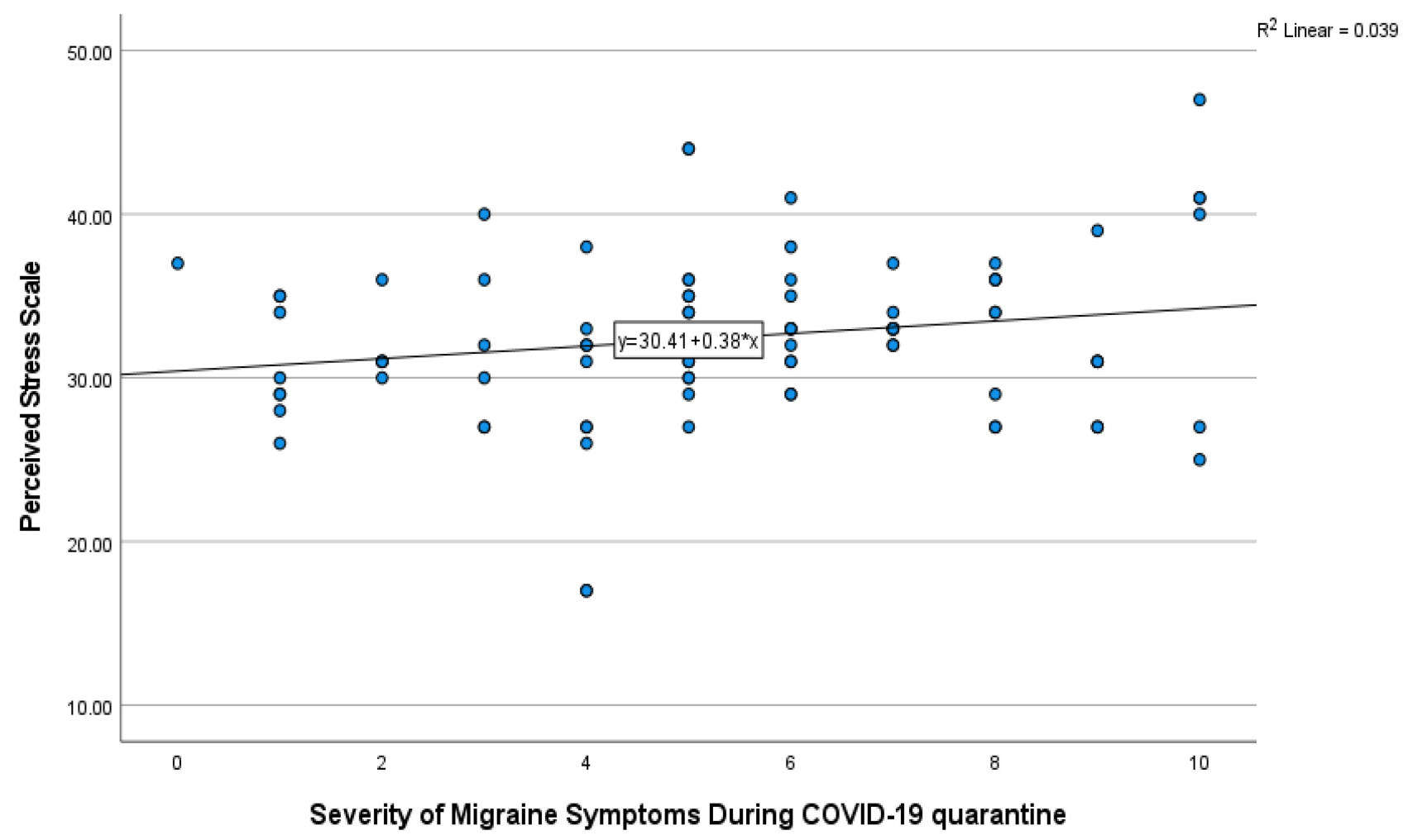

Figure 2 The relationship between the severity of migraine symptoms and stress during the COVID-19 quarantine. Abbreviation: COVID-19, coronavirus disease. 


\section{Data Sharing Statement}

All data generated or analyzed during this study are included in this published article.

\section{Ethics Approval and Consent to Participate}

All participants voluntarily in the study after receiving a clear description of the study objectives in an electronic consent form. They had the full right to withdraw from the study at any time. Ethical approval was obtained from the Institutional Review Board of Princess Nourah bint Abdulrahman University in late November 2020 before the data collection started in late December 2020. IRB Registration Number with KACST, KSA: H-01-R-059.

\section{Acknowledgments}

We would like to extend our gratitude to Dr. Amal Fayed for her help in all the steps of this research. Special thanks to the consultant of neurology Dr. Norah Alharbi for her help in our questionnaire design. We would like to acknowledge Princess Nourah Bint Abdulrahman University.

\section{Funding}

There was no cost for conducting this research. However, the cost of the publication will be provided by the Princess Nourah bint Abdulrahman University.

\section{Disclosure}

The authors declare that they have no conflicts of interest for this work.

\section{References}

1. Delussi M, Gentile E, Coppola G, et al. Investigating the effects of COVID-19 quarantine in migraine: an observational cross-sectional study from the Italian National Headache Registry (RICe). Front Neurol. 2020;11:1-8. doi:10.3389/fneur.2020.597881

2. Yang S, Hua M, Liu X, et al. COVID-19 treatment: close to a cure? A rapid review of pharmacotherapies for the novel coronavirus (SARS-CoV-2). Int $J$ Antimicrob Agents. 2020;56:106080. doi:10.1016/j.ijantimicag.2020.106080

3. World Health Organization. COVID-19 Dashboard. Geneva: world Health Organization; 2020. Available from: https://covid19.who.int/. Accessed December 2, 2021.

4. National Cancer Institute. Psychological stress and cancer; 2012. Available from: https://www.cancer.gov/about-cancer/coping/feelings/ stress-fact-sheet. Accessed June 29, 2021.
5. Headache Classification Committee of the International Headache Society (IHS) The International Classification of Headache Disorders. 3rd edition. Cephalalgia. 2018;38(1):1-211. doi:10.1177/ 0333102417738202

6. Deniz O, Aygül R, Koçak N, Orhan A, Kaya MD. Precipitating factors of migraine attacks in patients with migraine with and without aura. Pain Clin. 2004;16(4):451-456. doi:10.1163/ 1568569042664459

7. Ma M, Fang J, Li C, et al. The status and high risk factors of severe psychological distress in migraine patients during nCOV-2019 outbreak in Southwest China: a cross-sectional study. J Headache Pain. 2020;21(1):100. doi:10.1186/s10194-020-01168-5

8. Ibrahim NK, Alotaibi AK, Alhazmi AM, Alshehri RZ, Saimaldaher RN, Murad MA. Prevalence, predictors and triggers of migraine headache among medical students and interns in King Abdulaziz University, Jeddah, Saudi Arabia. Pak J Med Sci. 2017;33(2):270-275. doi:10.12669/pjms.332.12139

9. Wang S, Zhang Y, Ding W, et al. Psychological distress and sleep problems when people are under interpersonal isolation during an epidemic: a nationwide multicenter cross-sectional study. Eur Psychiatry. 2020;63(1):e77. doi:10.1192/j.eurpsy.2020.78

10. Parodi IC, Poeta MG, Assini A, Schirinzi E, Del Sette P. Impact of quarantine due to COVID infection on migraine: a survey in Genova, Italy. Neurol Sci. 2020;41(8):2025-2027. doi:10.1007/s10072-02004543-x

11. Al-Hashel JY, Ismail II. Impact of coronavirus disease 2019 (COVID-19) pandemic on patients with migraine: a web-based survey study. J Headache Pain. 2020;21(1):115. doi:10.1186/s10194020-01183-6

12. Gonzalez-Martinez A, Planchuelo-Gómez A, Guerrero AL, et al. Evaluation of the Impact of the COVID-19 Lockdown in the Clinical Course of Migraine. Pain Med. 2021;22(9):2079-2091. doi:10.1093/pm/pnaa449

13. Alkhamees AA, Alrashed SA, Alzunaydi AA, Almohimeed AS, Aljohani MS. The psychological impact of COVID-19 pandemic on the general population of Saudi Arabia. Compr Psychiatry. 2020;102:152192. doi:10.1016/j.comppsych.2020.152192

14. Lipton RB, Sadovsky R, Kolodner K, Endicott J, Hettiarachchi J, Harrison W. A self-administered screener for migraine in primary care: the ID Migraine validation study. Neurology. 2003;61:375-382. doi:10.1212/01.WNL.0000078940.53438.83

15. Karcioglu O, Topacoglu H, Dikme O, Dikme O. A systematic review of the pain scales in adults: which to use? Am J Emerg Med. 2018;36:707-714. doi:10.1016/j.ajem.2018.01.008

16. Alghadir AH, Anwer S, Iqbal ZA. The psychometric properties of an Arabic numeric pain rating scale for measuring osteoarthritis knee pain. Disabil Rehabil. 2016;38(24):2392-2397. doi:10.3109/ 09638288.2015.1129441

17. Chan SF, La Greca AM. Perceived Stress Scale (PSS). Encyclopedia Behav Med. 2020;1;1646-1648.

18. Mayo Clinic. Tension Headache - Symptoms and Causes - Mayo Clinic [Internet]. Mayo Clinic.; 2020.

19. Martin PR. Stress and primary headache: review of the research and clinical management. Curr Pain Headache Rep. 2016;20(45):1-8. doi:10.1007/s11916-016-0576-6

20. Gonzalez-Martinez A, Planchuelo-Gómez Á, Guerrero ÁL, et al. Effects of the onabotulinumtoxinA follow-up delay in migraine course during the COVID-19 lockdown. Neurol Sci. 2021:1-6. doi:10.1007/s10072-021-05180-8 


\section{Publish your work in this journal}

The Journal of Pain Research is an international, peer reviewed, open access, online journal that welcomes laboratory and clinical findings in the fields of pain research and the prevention and management of pain Original research, reviews, symposium reports, hypothesis formation and commentaries are all considered for publication. The manuscript management system is completely online and includes a very quick and fair peer-review system, which is all easy to use. Visit http:// www.dovepress.com/testimonials.php to read real quotes from published authors. 